\title{
Construções geométricas de polígonos regulares e aplicações à trigonometria
}

José Gilvan de Oliveira (iD

\author{
Moacir Rosado Filho (D)
}

Domingos Sávio Valério Silva (D)

\begin{abstract}
Resumo
No presente trabalho, apresentamos construções geométricas de alguns polígonos regulares, dentre eles, o pentágono e o decágono, usando apenas régua (não graduada) e compasso. A partir dessas construções, obtemos a célebre razão áurea e as razões trigonométricas dos ângulos $18^{\circ}, 36^{\circ}$ e $72^{\circ}$, que nos permitem ampliar os estudos em Trigonometria com novas aplicações envolvendo esses ângulos. Também apresentamos uma relação entre a construção do pentágono regular e a do icosaedro regular. Os ingredientes usados no texto são aqueles encontrados na Geometria Plana e são bem poucos, restritos a interseções de retas e circunferências, propriedades de triângulos e o Teorema de Pitágoras.
\end{abstract}

\begin{abstract}
In the presente work, we present geometric constructions of some regular polygons, among them, the pentagon and the decagon, by using only straightedge (ruler with no marks) and compass. From these constructions, we obtain the famous golden ratio and the trigonometric ratios of the angles $18^{\circ}, 36^{\circ}$ and $72^{\circ}$, which allow us to expand our studies in Trigonometry with new applications involving these angles. We also present a relationship between the construction of the regular pentagon and that of the regular icosahedron. The ingredients used in the text are those found in Basic Geometry and are very few, restricted to intersections of lines and circles, properties of triangles and also the Pythagorean Theorem.
\end{abstract}

Palavras-chave: Construções geométricas; Pentágono; Decágono; Razões trigonométricas.

\section{Introdução}

O objetivo principal deste artigo é abordar as construções do pentágono e do decágono regulares, juntamente com o cálculo das principais razões trigonométricas dos correspondentes ângulos centrais. Nos livros didáticos, os exemplos e exercícios envolvendo razões trigonométricas são normalmente restritos aos chamados $\operatorname{arcos}$ notáveis dos ângulos $30^{\circ}, 45^{\circ}$ e $60^{\circ}$. Embora esses ângulos não sejam o objetivo principal do presente artigo, eles serão abordados porque os ingredientes usados nesses casos são exatamente os mesmos que usaremos para construir o pentágono e o decágono regulares. A diferença para as construções desses dois últimos fica restrita apenas ao número de etapas necessárias durante todo o processo de construção. Assim procedendo, pretendemos mostrar ao leitor que é possível ampliar o estudo da Trigonometria, abordando novas aplicações e exercícios, por exemplo, com os ângulos $18^{\circ}, 36^{\circ}$ e $72^{\circ}$. Os ingredientes usados no texto são aqueles encontrados na Geometria Plana e são bem poucos, restritos a interseções de retas e circunferências, propriedades de triângulos e o Teorema de Pitágoras. 
Para cada número inteiro $\mathrm{n} \geq 3$, seja $\mathscr{P}_{\mathrm{n}}$ um polígono regular de $\mathrm{n}$ lados inscrito em uma circunferência de raio unitário e centro $\mathrm{O}$, considerados em um plano estabelecido. Sejam $\ell_{\mathrm{n}}$ o comprimento do lado de $\mathscr{P}_{\mathrm{n}}$ e seja $\theta_{\mathrm{n}}$ o ângulo central interno em $\mathrm{O}$ de um triângulo com vértices em $\mathrm{O}$ e dois vértices consecutivos de $\mathscr{P}_{\mathrm{n}}$. Então, como a circunferência de raio unitário está dividida em $\mathrm{n}$ arcos de mesma medida, o ângulo central $\theta_{\mathrm{n}}$ é dado por $\theta_{\mathrm{n}}=360^{\circ} / \mathrm{n}$, e, daí, conforme a Figura 1 , temos sen $\left(\theta_{\mathrm{n}} / 2\right)=\ell_{\mathrm{n}} / 2$ e, portanto, $\operatorname{sen}\left(180^{\circ} / \mathrm{n}\right)=\ell_{\mathrm{n}} / 2$. Uma questão que vamos estudar é como efetivamente dividir a circunferência unitária dessa forma, ou seja, em n partes iguais. O objetivo principal desse artigo é apresentar resposta para os casos $\mathrm{n}=5$ e $\mathrm{n}=10$, isto é, construir o pentágono regular $\mathscr{P}_{5}$ e o decágono regular $\mathscr{P}_{10}$. Faremos isso repetindo as mesmas técnicas dos dois casos precedentes $n=3$ e $n=4$. Como consequência diretamente relacionada com a construção do pentágono regular, obteremos também os casos $n=10$ e $n=20$.

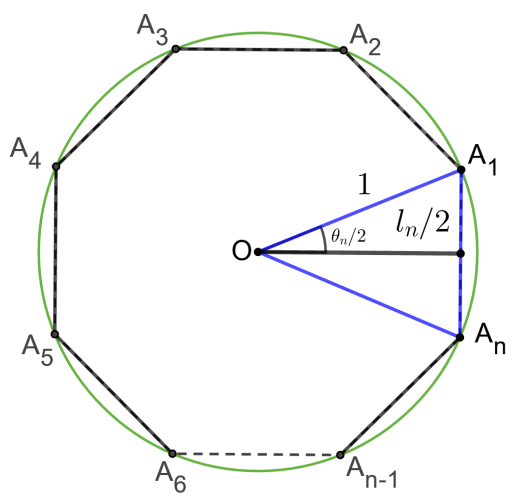

Figura 1: Polígono regular de n lados

\subsection{Caso $n=3$}

Neste caso, temos $\theta_{3}=360^{\circ} / 3=120^{\circ}$ e $\operatorname{sen}\left(\theta_{3} / 2\right)=\operatorname{sen} 60^{\circ}=\ell_{3} / 2$. Considere a Figura 2 abaixo. Dado o segmento de reta $\mathrm{AA}^{\prime}$, determinamos o seu ponto médio $\mathrm{O}$, obtido a partir da interseção das duas circunferências de centros nas extremidades do segmento $\mathrm{AA}^{\prime}$ e mesmo raio $\mathrm{r}$ maior ou igual à metade do comprimento de $\mathrm{AA}^{\prime}$. Os dois triângulos com lado comum OA são isósceles e congruentes, pelo caso LLL de congruência de triângulos. De modo análogo, considerando o segmento OA, obtemos seu ponto médio C. Além disso, $\mathrm{C}$ é o ponto médio do segmento $\mathrm{BB}^{\prime}$. Em particular, se $\mathrm{r}=\mathrm{OA}$, então os dois triângulos são equiláteros, a medida do ângulo $\alpha$ é $60^{\circ}$ e os dois triângulos com lado comum OC são congruentes, pelo caso LAL. Portanto, eles são também triângulos retângulos. Finalmente, como temos $\alpha$ como um ângulo externo do triângulo $\mathrm{A}^{\prime} \mathrm{OB}$, a medida dos ângulos da base desse triângulo isósceles é $\alpha / 2=30^{\circ}$. A mesma conclusão vale para o triângulo $\mathrm{A}^{\prime} \mathrm{OB}^{\prime}$. Isso permite concluir que $\mathrm{A}^{\prime} \mathrm{B}^{\prime} \mathrm{B}$ é um triângulo equilátero e, logo, o ângulo $\angle(\mathrm{OBC})$ é igual a $\alpha / 2=30^{\circ}$. Além disso, considerando o segmento OA unitário, pelo Teorema de Pitágoras aplicado ao triângulo retângulo $\mathrm{OCB}$, concluímos então que sen $30^{\circ}=1 / 2$, sen $60^{\circ}=\sqrt{3} / 2 \mathrm{e}$ que o lado $\ell_{3}$ do triângulo equilátero $\mathrm{A}^{\prime} \mathrm{B}^{\prime} \mathrm{B}$ é $\ell_{3}=\sqrt{3}$. 


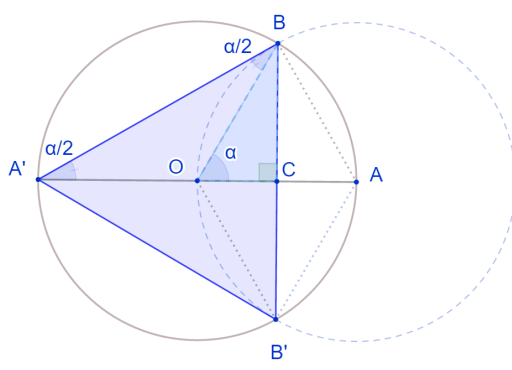

Figura 2: Construção do triângulo equilátero

Vale destacar que o procedimento descrito, tomando-se a interseção de duas particulares circunferências, permite efetivamente dividir uma circunferência em três partes iguais e, assim, construir o polígono regular $\mathscr{P}_{3}$. O processo pode ser resumido em duas etapas: a) Dado o segmento $\mathrm{AA}^{\prime}$ determinamos seu ponto médio $\mathrm{O}$; b) Em seguida, a partir de $\mathrm{O}$ determinamos os pontos $\mathrm{B}$ e $\mathrm{B}^{\prime}$ de interseção da mediatriz do segmento $\mathrm{OA}$ com a circunferência de centro $\mathrm{O}$ e raio $\mathrm{OA}$. Outro destaque é que o método permite efetivamente obter a mediatriz de um segmento qualquer e, portanto, bissetriz, mediana e altura, em relação à base, de um triângulo isósceles qualquer. Isso será usado nos outros casos considerados a seguir. No caso da Figura 2 temos esses conceitos destacados, por exemplo, tanto para o triângulo $\mathrm{OAB}$ quanto para o triângulo $\mathrm{A}^{\prime} \mathrm{B}^{\prime} \mathrm{B}$. Assim, o ponto $\mathrm{C}$ é ponto médio com relação ao segmento $\mathrm{OA}$, bem como com relação ao segmento BB'.

\subsection{Caso $n=4$}

Neste caso, temos $\theta_{4}=360^{\circ} / 4=90^{\circ}$ e $\operatorname{sen}\left(\theta_{4} / 2\right)=\operatorname{sen} 45^{\circ}=\ell_{4} / 2$. Considere a Figura 3 abaixo.

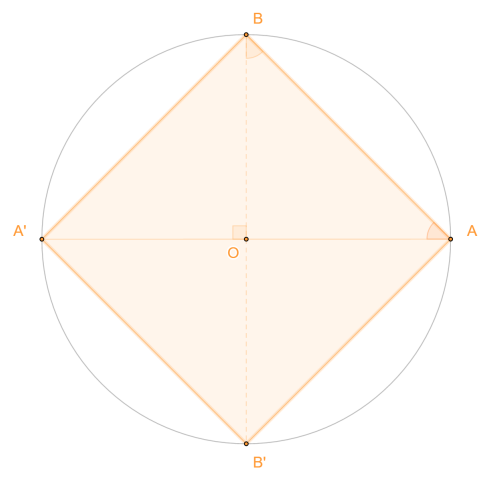

Figura 3: Construção do quadrado

Dado o segmento de reta $\mathrm{AA}^{\prime}$, seguindo o procedimento descrito no caso anterior, determinamos o seu ponto médio $\mathrm{O}$. Em seguida determinamos os pontos de interseção $\mathrm{B}$ e $\mathrm{B}^{\prime}$ da mediatriz de $\mathrm{AA}^{\prime}$ com a circunferência de centro no ponto $\mathrm{O}$ e raio $\mathrm{OA}$. Obtemos assim o triângulo retângulo AOB. Como esse 
triângulo é também isósceles, considerando OA como sendo unitário e usando o Teorema de Pitágoras, obtemos assim de maneira efetiva o quadrado inscrito $\mathscr{P}_{4}$. Além disso, sen $45^{\circ}=\sqrt{2} / 2$ e $\ell_{4}=\sqrt{2}$.

\section{Pentágono e Decágono Regulares}

\subsection{Caso $n=5$}

Neste caso, temos $\theta_{5}=360^{\circ} / 5=72^{\circ}$ e sen $\left(\theta_{5} / 2\right)=\operatorname{sen} 36^{\circ}=\ell_{5} / 2$. Daí, a partir da soma dos ângulos internos de um triângulo e das igualdades $180^{\circ}=36^{\circ}+2 \times 72^{\circ}$ e $180^{\circ}=36^{\circ}+\left(36^{\circ}+36^{\circ}\right)+72^{\circ}$, isso sugere a existência de dois triângulos isósceles semelhantes com bases $\ell$ e $1-\ell$, como na Figura 4 abaixo, onde $\alpha=36^{\circ}$. Tais triângulos serão posteriormente obtidos de maneira efetiva e, nesse caso, $\ell$ é o lado $\ell_{10}$ do polígono regular $\mathscr{P}_{10}$. Segue, da semelhança desses triângulos, que $\ell=\ell / 1=(1-\ell) / \ell$, e, portanto, $\ell=(\sqrt{5}-1) / 2$, que é a célebre razão áurea ([1] e [4]).

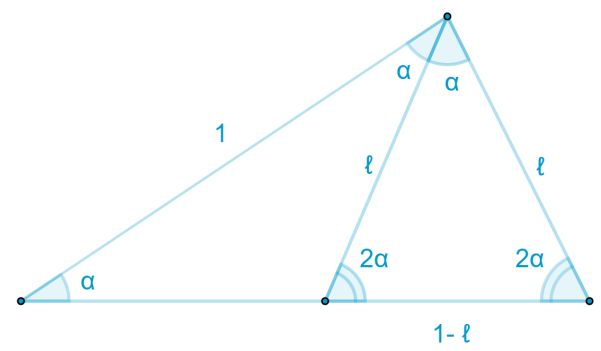

Figura 4: Razão áurea

Com conhecimento desses fatos, vamos construir a seguir, de forma efetiva, o polígono regular $\mathscr{P}_{5}$, ou seja, o pentágono regular. Considere a Figura 5 abaixo.

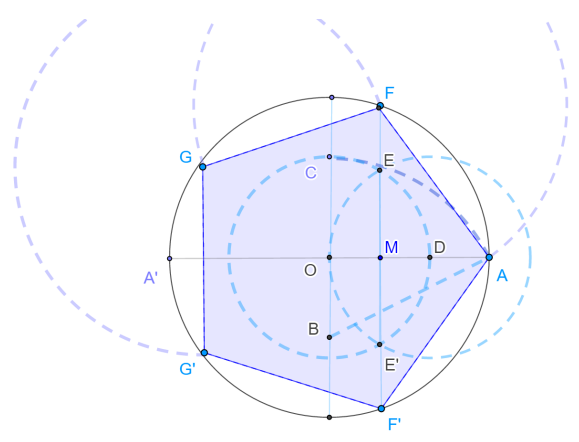

Figura 5: Construção do pentágono regular

Dado o segmento de reta $\mathrm{AA}^{\prime}$ determinamos, a partir do procedimento detalhado no primeiro caso tratado $(\mathrm{n}=3)$, sua mediatriz contendo o ponto médio $\mathrm{O}$ de $\mathrm{AA}^{\prime}$ e também a circunferência com centro $\mathrm{O}$ e raio 
OA. Em seguida, determinamos o ponto B da mediatriz mencionada de forma que OB é a metade do raio da circunferência. Admitindo-se que o segmento OA seja unitário, pelo Teorema de Pitágoras aplicado ao triângulo retângulo $\mathrm{AOB}$, temos $\mathrm{AB}=\sqrt{5} / 2$. Portanto, $\mathrm{OC}=\mathrm{AB}-1 /(2=((\sqrt{5}-1)) / 2)$, que é a razão áurea, onde $\mathrm{C}$ é um dos pontos de interseção da mesma mediatriz com a circunferência com centro $\mathrm{B}$ e raio $\mathrm{AB}$. O ponto $\mathrm{D}$ é a interseção do segmento $\mathrm{OA}$ com a circunferência de centro $\mathrm{O}$ e raio OC. Finalmente, os

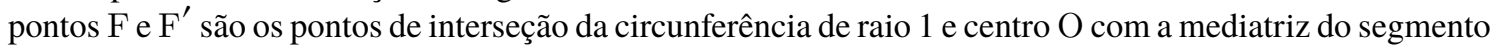
OD. Como o triângulo OFD é isósceles, com dois lados unitários e com base igual à razão áurea, então ele é congruente ao triângulo isósceles da Figura 4, com ângulos da base $\ell$ iguais a $72^{\circ}$. Assim, os pontos $\mathrm{F}^{\prime}, \mathrm{A} \mathrm{e}$ F são três pontos consecutivos de $\mathscr{P}_{5}$. Os pontos restantes de $\mathscr{P}_{5}$ são os pontos $\mathrm{G}$ e $\mathrm{G}^{\prime}$ obtidos da interseção da circunferência unitária de centro $\mathrm{O}$ com as circunferências de mesmo raio $\mathrm{AF}$ e centros nos pontos $\mathrm{F}$ e $\mathrm{F}^{\prime}$. Além disso, como os ângulos da base do triângulo isósceles OFD são iguais a $72^{\circ}$ e a altura FM desse triângulo em relação ao vértice $\mathrm{F}$ é a metade de $\mathrm{FF}^{\prime}$, segue do Teorema de Pitágoras que

$$
\begin{gathered}
\operatorname{sen} 72^{\circ}=\mathrm{FM}=\sqrt{(5+\sqrt{5}) / 8}=\sqrt{(10+2 \sqrt{5})} / 4, \\
\mathrm{FF}^{\prime}=\sqrt{(10+2 \sqrt{5})} / 2,
\end{gathered}
$$

e, usando a bissetriz do triângulo OFD em relação ao vértice $\mathrm{F}$, temos

$$
\operatorname{sen} 18^{\circ}=\mathrm{OM} / \mathrm{OF}=(\sqrt{5}-1) / 4
$$

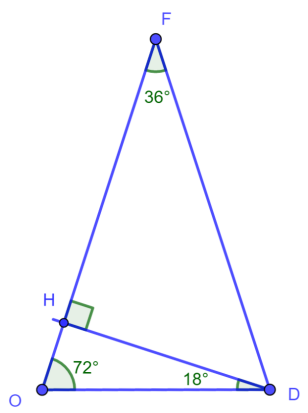

Figura 6: Razão áurea

Em seguida, calculando a altura do triângulo OFD em relação ao vértice D por meio do Teorema de Pitágoras (veja Figura 6), obtemos

$$
\operatorname{sen} 36^{\circ}=\mathrm{DH} / \mathrm{DF}=\sqrt{(5-\sqrt{5}) / 8}=\sqrt{(10-2 \sqrt{5})} / 4
$$

Daí, os lados do pentágono regular $\mathscr{P}_{5}$ e do decágono regular $\mathscr{P}_{10}$ são, respectivamente,

$$
\ell_{5}=\sqrt{(10-2 \sqrt{5})} / 2 \text { e } \ell_{10}=(\sqrt{5}-1) / 2 \text {. }
$$


Outra propriedade interessante a ser destacada do pentágono regular, como se pode verificar das informações acima, é a razão áurea como quociente do comprimento do lado pela diagonal do pentágono regular. Em particular, usando o caso LLL de semelhança de triângulos, se o lado AF na Figura 5 é 1, então a diagonal FF' é $(1+\sqrt{5}) / 2(\operatorname{Ver}[4])$.

A partir dos resultados anteriores e da relação $\cos ^{2} \theta+\operatorname{sen}^{2} \theta=1$, obtemos as tabelas.

\begin{tabular}{|c|c|c|c|}
\hline$\theta$ & $30^{\circ}$ & $45^{\circ}$ & $60^{\circ}$ \\
\hline \hline $\operatorname{sen} \theta$ & $1 / 2$ & $\sqrt{2} / 2$ & $\sqrt{3} / 2$ \\
\hline $\cos \theta$ & $\sqrt{3} / 2$ & $\sqrt{2} / 2$ & $1 / 2$ \\
\hline
\end{tabular}

\begin{tabular}{|c|c|c|c|}
\hline$\theta$ & $18^{\circ}$ & $36^{\circ}$ & $72^{\circ}$ \\
\hline \hline $\operatorname{sen} \theta$ & $\frac{\sqrt{5}-1}{4}$ & $\frac{\sqrt{10-2 \sqrt{5}}}{4}$ & $\frac{\sqrt{10+2 \sqrt{5}}}{4}$ \\
\hline $\cos \theta$ & $\frac{\sqrt{10+2 \sqrt{5}}}{4}$ & $\frac{\sqrt{5}+1}{4}$ & $\frac{\sqrt{5}-1}{4}$ \\
\hline
\end{tabular}

Exemplo 1. O Plano Diretor de certo município litorâneo, para garantir a adequada insolação da praia em certa data e horário do ano, estabelece que a altura das edificações não poderá ultrapassar o ângulo de $36^{\circ}$, medido a partir do calçadão segundo uma linha horizontal, até o ponto mais elevado da fachada, conforme a Figura 7 abaixo. Admitindo que a distância do calçadão até a base de uma edificação é igual a 21 metros, qual é a altura máxima h da edificação?

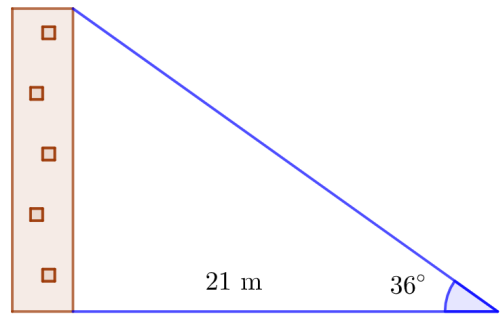

Figura 7

Solução: Para a altura máxima h da edificação, temos

$$
h / 21=\operatorname{tg} 36^{\circ}=\operatorname{sen} 36^{\circ} / \cos 36^{\circ}=\sqrt{(10-2 \sqrt{5})} /(1+\sqrt{5}) .
$$

Logo, $h \cong 15,25$. Portanto, a edificação deverá ter altura máxima de aproximadamente 15,25 metros. Considerando que, na construção civil cada pavimento tem altura média de 3 metros, essa edificação corresponde a um prédio de aproximadamente 5 pavimentos. 
Retornando à construção do pentágono regular aqui apresentada, vemos que o ponto $\mathrm{C}$ na Figura 5 é um dos pontos de interseção da reta contendo os pontos $\mathrm{B}$ e $\mathrm{O}$ com a circunferência de centro $\mathrm{B}$ e raio igual a AB. Considerando então o outro ponto de interseção, digamos C', obtemos o chamado retângulo áureo determinado pelos vértices consecutivos $\mathrm{A}, \mathrm{O}$ e C', com medida dos lados 1 e $((1+\sqrt{5})) / 2$ (Ver [1], [4]). Um destaque importante desse retângulo, com relação à construção do pentágono regular, é a construção de Luca Pacioli para o icosaedro regular, descrita a seguir (Ver [3], p. 8). Ela é obtida a partir da interseção de três retângulos áureos perpendiculares, dispostos em particular posição de simetria destacada na Figura 8a. Para cada um dos doze vértices desses retângulos áureos perpendiculares existem outros cinco vértices que, juntamente com o vértice inicial, formam cinco triângulos equiláteros. Mais ainda, esses cinco pontos são coplanares e determinam um pentágono regular. O poliedro determinado por todos os doze vértices é o icosaedro regular (Figura 8b), que é um dos cinco poliedros regulares, únicos existentes, e que foram todos descobertos pelos matemáticos gregos antigos.

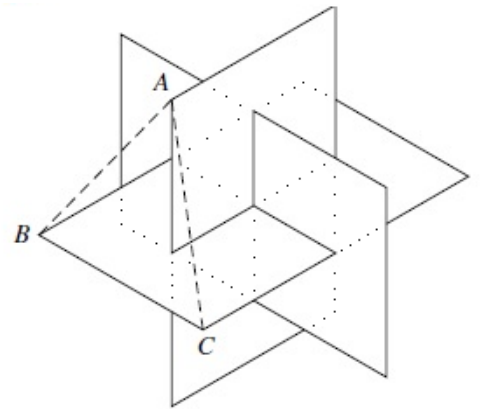

Figura 8a: Retângulos áureos perpendiculares

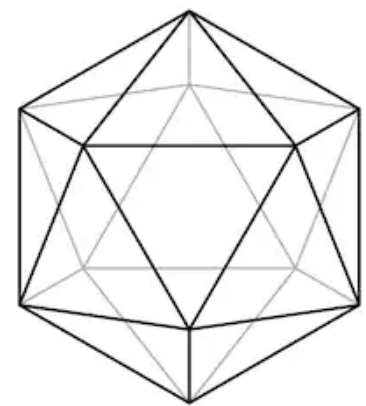

Figura 8b: Icosaedro regular

\section{Conclusão}

No caso tratado do triângulo equilátero, Figura 2, foi tomada a mediatriz do segmento OA. Um procedimento análogo pode ser feito tomando a mediatriz de $\mathrm{A}^{\prime} \mathrm{O}$. Esses dois procedimentos simultâneos produzem o hexágono regular $\mathscr{P}_{6}$. Da mesma forma, no caso do pentágono regular, Figura 5, o ponto $\mathrm{D}$ foi tomado no segmento $\mathrm{OA}$ dentre as quatro possíveis interseções da circunferência de centro $\mathrm{O}$ e raio $\mathrm{OC}$. O mesmo procedimento poderia ser feito usando a interseção com A' O. Essas duas escolhas simultâneas produzem o decágono regular $\mathscr{P}_{10}$, enquanto todas as quatro alternativas simultâneas produzem o icoságono regular $\mathscr{P}_{20}$. Cada um desses casos leva-nos às razões trigonométricas relativas aos ângulos $72^{\circ}, 36^{\circ}$, e $18^{\circ}$, assim como ao ângulo $54^{\circ}$, que é o ângulo complementar de $36^{\circ}$. Mais ainda, como estímulo ao leitor, lembrando que um ângulo inscrito em uma circunferência é a metade do seu correspondente ângulo central (Figura 2), temos até mesmo a construção do ângulo $9^{\circ}$ e do seu complementar $81^{\circ}$, enriquecendo ainda mais as possibilidades de aplicações. Também, usando as fórmulas de adição, é possível calcular as razões trigonométricas do ângulo $3^{\circ}$, a partir das razões trigonométricas dos ângulos $18^{\circ}, 30^{\circ}$ e $45^{\circ}$.

Uma curiosidade natural é saber o que ocorre sobre a efetiva construção de $\mathscr{P}_{\mathrm{n}}$ se $\mathrm{n}$ é maior do que 5 . Podemos separar a questão em duas situações, dependendo da paridade de $\mathrm{n}$. Se $\mathrm{n}$ é par, digamos $\mathrm{n}=2 \mathrm{k}$, então o método usado anteriormente, considerando a mediatriz do lado $\ell_{\mathrm{k}}$, pode ser aplicado admitindo-se que já é conhecido o resultado para o polígono regular $\mathscr{P}_{\mathrm{k}}$. Caso contrário, se n é impar, então já para $\mathrm{n}=7$ é conhecido que o método não pode ser aplicado. No caso geral, uma resposta foi dada por Gauss, dependendo da fatoração de $n$ em números primos: $\mathscr{P}_{n}$ é construtível se, e somente, se $n=2^{r} \times p_{1} \times \ldots \times p_{k}$, onde $r \in \mathbb{N}$ e $\mathrm{p}_{1}, \ldots, \mathrm{p}_{\mathrm{k}}$ são distintos primos da forma $\mathrm{p}_{\mathrm{i}}=2^{2^{\mathrm{s}_{\mathrm{i}}}}+1,1 \leq \mathrm{i} \leq \mathrm{k}, \mathrm{s}_{\mathrm{i}} \in \mathbb{N}$ (veja por exemplo [2]). Os 
números primos dessa forma são conhecidos como números primos de Fermat. São conhecidos apenas os cinco primeiros primos de Fermat, a saber: 3, 5, 17, 257 e 65.537. Sabe-se que vários números da forma $2^{2^{\mathrm{n}}}+1, \mathrm{n} \in \mathbb{N}$, não são primos. Por exemplo, Euler foi a primeira pessoa a alertar sobre isso, mostrando que o número $2^{2^{5}}+1$ tem 641 como fator primo e, logo, não é primo.

\section{Referências}

[1] ÁVILA, G.S.S. "Retângulo áureo, divisão áurea e sequência de Fibonacci”. RPM 6, 1985.

[2] GONÇALVES, A. Introdução à Álgebra. Coleção Projeto Euclides, SBM, 2017.

[3] STILLWELL, J. Elements of Algebra - Geometry, Numbers, Equations. Undergraduate Texts in Mathematics, Springer, 2001.

[4] WAGNER, E. “O Símbolo da SBM”. RPM 20, 1992.

José Gilvan de Oliveira

DMAT/UFES

<jgilvanol@gmail.com>

Moacir Rosado Filho

DMAT/UFES

<moacir@ele.ufes.br>

Domingos Sávio Valério Silva

DMAT/UFES

<dsvaleriosilva@gmail.com>

Recebido: 23/09/2020

Publicado: 21/05/2021 